\title{
Habitat deterioration, signals and conservation
}

\section{Candolin, Ulrika}

Elsevier Scientific Publ. Co

2019-01

Candolin , U 2019 , Habitat deterioration, signals and conservation . in J Choe (ed.), Encyclopedia of Animal Behaviour . 2nd Edition edn , vol. 1 , Elsevier Scientific Publ. Co, pp. 266-272 . https://doi.org/10.1016/B978-0-12-809633-8.01126-2

http://hdl.handle.net/10138/261740

https://doi.org/10.1016/B978-0-12-809633-8.01126-2

acceptedVersion

Downloaded from Helda, University of Helsinki institutional repository.

This is an electronic reprint of the original article.

This reprint may differ from the original in pagination and typographic detail.

Please cite the original version. 


\title{
Habitat deterioration, signals and conservation
}

\author{
Ulrika Candolin \\ Dept of Biological \& Environmental Sciences \\ University of Helsinki, Finland
}

\begin{abstract}
Changes in the environment because of human activities can influence signals and thereby animal communication. The changes can hamper the transmission and reception of the signals, or disrupt the link between the signals and the information they should convey, resulting in dishonest signaling. When habitat deterioration alters signals used in mate choice, maladaptive mate choices may result that reduce the viability of future generations. This can cause population declines and decrease biodiversity, and, hence, be of conservation concern. However, little is currently known about the long-term consequences of disturbed communication for animal populations and community structure.
\end{abstract}

\section{Keywords}

Adaptation, Communication, Environmental change, Evolutionary trap, Fitness, Honest advertisement, Male-male competition, Mate choice, Phenotypic plasticity, Sexually selected traits, Sexual selection, Signal design and Signal transmission

\section{Glossary}

Adaptation The process, driven by natural selection, whereby species or populations become better suited to the environment. It takes place over generations and results in an increase in those genes (or alleles) that allow individuals in a population to better survive and reproduce in an environment.

Evolutionary trap Occurs when environmental change renders formerly adaptive cues maladaptive and this causes organisms to make maladaptive behavioral or life-history decisions. Organisms are then 'trapped' by their evolutionary response to the cue.

Fitness The fitness of an individual is the proportion of the individual's genes, or more correctly alleles, that are passed on to the following generations.

Phenotypic plasticity The adjustment of the phenotype (the observable characteristics of an organism) to changes in the environment. Plasticity has a genetic basis that determines how an individual responds to the change, ie, the genetically determined reaction norm.

Reaction norm The range of phenotypes expressed by a single genotype across different environments.

Sexual signals Advertise the signaler's genetic or phenotypic quality. The signals are used to attract mates and deter rivals. Examples include conspicuous traits, such as bright colors and elaborate songs. Signals can be visual, acoustic, olfactory, tactile or electric.

\section{Introduction}

For animal communication to be effective, signals must be efficiently transmitted and the information they convey reliable. The ongoing human-caused deterioration and destruction of habitats is altering the conditions required for efficient communication. This is influencing particularly mate choice, as the choice of a suitable mate is often based on the evaluation of a range 
of signals that are assumed to reflect mate quality. Yet, little is known about the consequences that disturbed communication during mate choice have for populations. It could alter the number and viability of individuals born into a population, which could endanger its persistence and, hence, be of conservation concern.

Here, I discuss what is known about the effects of habitat deterioration on signals and communication during mate choice, as well as the further consequences that altered communication can have for population viability. I focus on major human-induced changes that are known to alter communication, such as changed visibility, increased noise levels and the discharge of chemicals. In particular, I explain the consequences that altered signals and communication could have for individuals and populations, and, ultimately, for biodiversity and the structure and function of species communities.

\section{Why Use Signals?}

Many species use signals to attract mates and deter rivals, such as bright colors, loud vocalizations and vigorous courtship displays. The signals may have no other function than to increase mating success and, hence, are often sexually selected. They evolve when their benefit in improving lifetime mating success is higher than their cost. They may deter rivals by reflecting fighting ability, or attract mates by indicating some quality essential for increasing the number or quality of offspring produced, such as parenting skills, or the genetic quality of the signaler, which can be inherited by the offspring. For example, male barn swallows (Hirundo rustica) show off their tail feathers during an elaborate courtship display towards females, and the length of these feathers reflects male viability. Consequently, females prefer males with longer tail feathers, which results in males with long feathers acquiring mates sooner and having a higher reproductive success than males with shorter feathers (M øller, 1994).

Typical costs of conspicuous signals that constrain their evolution are increased energy expenditure - because of the development and expression of the signals - and the attraction of unwanted receivers, such as predators. These costs often increase with signal elaboration, so that only individuals in the best condition can afford to develop the most elaborate signals. This ensures that signal elaboration reflects mate quality, ie, that the advertisement of mate quality is honest and that mate choosers benefit from paying attention to the signals.

Over evolutionary time, signals are expected to evolve to an optimum expression level where benefits are balanced against costs so that the net benefit of signaling is maximized. This optimum depends on environmental conditions. Changes in the environment that alter the optimum signal expression can result in maladaptive mate choices with negative effects on offspring production.

\section{Why Are Signals Environment-Dependent?}

The production, transmission and reception of signals depend on environmental conditions, such as visibility and noise levels. Species that occupy different habitats are therefore expected to vary in their signal design. An example is males of a species pair of lizards, Anolis cooki and Anolis cristatellus, that use a throat fan, a colorful dewlap, to attract females and repel competing males from their territory. The two species occupy distinct local light environments and therefore differ in the color of their dewlap. The difference increases the contrast of the dewlap to respective background, which enhances the transmission of the signal in each habitat (Leal and Fleishman, 2004).

How sensitive signals are to environmental change depends on the complexity of the signal display. More complex displays that contain several information rich components convey more information 
than simpler displays consisting of only a single or a few components. However, they also suffer higher rates of environmental attenuation and, hence, are more sensitive to environmental change. Thus, species with complex displays are often more severely affected by habitat deterioration than species with simpler displays.

To signal efficiently in a changing environment, individuals need to adjust their signals to the changes that occur in the environment. How they do this, and what it requires, are discussed in the next section.

\section{Adjusting Signals to Environmental Change}

There are two main pathways by which signals can be adjusted to changes in the environment. The first is through phenotypic plasticity, in which case signalers adjust their signals to the environment according to their genetically determined reaction norms. The other possibility is genetic (evolutionary) changes, in which case selection over successive generations gradually adapts the population to the new conditions. In the first case, changes in signals are visible within a generation, sometimes immediately, in the latter case, in future generations and often after a considerable time lag.

When the environmental change occurs gradually, or the effect on the viability of the population is minor, species may be able to progressively evolve signals better adapted to the new conditions. For instance, a gradual increase in the carotenoid content of the food can reduce the value of red colors as indicators of foraging ability, as most individuals will then acquire enough carotenoids to develop bright red colors. The use of the color in mate choice and competition for mates may then decrease, which can relax selection for bright red ornaments and result in the evolution of duller coloration. The species may instead evolve other signals, such as other colors, or signals in other modalities, such as auditory signals.

If the environmental change proceeds more quickly than the speed at which signals can evolve, then signals have to be plastically adjusted to the change to continue to be effective. For example, a sudden increase in predation risk may result in males spending less time expressing conspicuous signals. Such adjustments require adaptive reaction norms for how to respond to the changes. However, these norms have evolved under previous conditions and need not be adaptive under altered conditions. Yet, plastic alterations may be the only possible response to rapid environmental change, particularly in species with longer generation time, such as larger vertebrates. Moreover, because species with longer generation time often have highly conspicuous mating signals, due to their low fecundity and need for careful mate choice, they can be highly sensitive to human-induced environmental change.

When the environmental change occurs faster than evolution can proceed, and adaptive reaction norms do not exist, the cost of signaling may increase, or the benefit of using the signals decrease. For instance, the signals may become more difficult to detect or judge, or the link between the signals and the sought benefits may be disrupted, resulting in dishonest signaling. Such changes can result in mal-adaptive mate choices. Mal-adaptive mate choices can in turn reduce the number and fitness of individuals born into the population and, hence, cause population decline.

In the following sections, I will discuss the influence that various environmental changes have on signals and communication during mate choice, including altered visibility, noise and chemical pollution.

\subsection{Visibility}

Vision is an important sensory channel for many animals. An efficient transmission and reception of visual signals depend on a range of factors, such as light conditions, background properties, the attenuation and degradation of the signals during transmission, and the sensory properties of the receiver. Humans are currently altering these factors through various activities, such as pollution and 
land use, and, hence, often have negative impacts on the efficacy of visual signals. This is affecting the ability of animals to attract mates and make careful mate choices and, hence, increasingly causing mal-adaptive mate choices.

Changes in the habitat that alter the contrast of visual signals against the background are particularly likely to hamper signal detection. For instance, land use that transforms habitats from lush green vegetation to barren soil reduces the conspicuousness of red ornamental signals. Similarly, changes in background movements hamper the detection of body movements, such as courtship displays. For example, increased wind speed hinders the detection of animals courting among wind-blown plants, which decreases mate encounter rate and thereby the opportunity for mate choosiness.

A common cause of reduced visibility in aquatic environments is altered primary production, as this can hamper the transmission of visual signals through the medium. For instance, human-induced growth of macroalgae and phytoplankton reduces visibility and alters the use of visual signals. An example is the three-spined stickleback (Gasterosteus aculeatus) in the Baltic Sea, which suffers from deteriorating visibility because of an increased growth of filamentous algae and extensive micro-algal blooms (Candolin, 2009). Visual communication is important in the species, as males develop red nuptial coloration to attract females and deter rivaling males, and the color is exposed through a conspicuous courtship dance towards females and during aggressive attacks on competing males (Fig. 1). To counteract the negative effect of reduced visibility in female attraction, males enhance their courtship activity. However, this increases the time and energy spent on courtship and, hence, the cost of mate attraction (Fig. 1). Similarly, females spend more time assessing males when visibility is poor, which increases their cost of mate evaluation (Fig. 1). Interestingly, females increase their use of olfactory signals when visibility deteriorates, which suggests that they might attempt to compensate for the impaired ability to use visual cues (Heuschele et al., 2009). However, mate choice is still altered, which suggests that some crucial information is lost when visual signals become more difficult to judge.

\subsection{Noise}

Anthropogenic noise arising from urbanization and traffic is a major global pollutant that hampers the transmission of auditory signals and, hence, interferes with acoustic communication. This is troublesome for species that rely on sound for detection and evaluation of mates. For example, great tits (Parus major) suffer from their songs being masked by human-induced low frequency noise in urban areas. To prevent the masking, birds have to sing with a higher minimum frequency (Slabbekoorn and Peet, 2003). However, all birds are not able to alter their songs, and these consequently suffer from impaired communication in noisy habitats.

In aquatic environments, underwater noise pollution from shipping is interfering with communication and mate choice. For instance, the noise from ferry boats increases the auditory threshold of the Lusitanian toadfish (Halobatrachus didactylus), which hampers its ability to detect conspecific acoustic signals (Vasconcelos et al., 2007). This influences the reproductive success of males, as they use the signals both during agonistic interactions and in the attraction of females. Similarly, vessel noise restricts acoustic communication in humpback whales (Megaptera novaeangliae). Worryingly, the whales cannot cope with the nose by modifying their sounds, as they can with natural noise, such as wind-dominated noise, which renders them highly sensitive to the noise pollution (Dunlop, 2016).

Anthropogenic noise is a mounting human-induced problem with the growth of the human population and the spread of urbanization and human activities around the globe. The degree to which animals will be able to adjust their acoustic communication to this rising problem, through plastic alterations or genetic changes, is poorly known. Sublethal impacts of noise have the potential to profoundly alter interactions both within and between species, and thereby decrease the abundance of species that cannot adjust their acoustic communication to noise. 


\subsection{Chemical Pollution}

Chemical communication is the most widely used sensory modality in nature. Thus, anthropogenic activities that pollute habitats with various chemicals is of great concern. For instance, the influx of untreated sewage and agricultural waste is changing the chemical environment of water bodies, which influences the production, reception and processing of chemical signals. Thus, species that rely on olfactory signals during mate searching and mate evaluation may have their mate choices altered. An example is the swordtail fish Xiphophorus birchmanni that is exposed to sewage effluent and agricultural runoff in parts of its distribution. The pollution elevates the concentration of humid acids in the water, which removes the ability of females to recognize male conspecifics. This results in hybridizations with related species (Fisher et al., 2006).

Another human induced problem is the acidification of oceans. The current increase in the concentration of carbon dioxide in the atmosphere has decreased the saturation of oceans with calcium carbonate, which in turn has reduced water $\mathrm{pH}$. Because acidification of water changes the value and quality of olfactory signals, mate choice can be altered. For instance, several fishes are less able to detect chemical signals when $\mathrm{pH}$ is reduced (Sundin et al., 2013).

A further growing problem, with potentially devastating effects on communication, is the increased release of endocrine disrupting chemicals into nature. This influences the expression of sexual signals as well as the ability of females to evaluate signals and respond to them in an adaptive way (Shenoy and Crowley, 2011). For instance, males of the palmate newt (Triturus helveticus) reduce the expression of body ornaments (tail height and hind-foot web area) when exposed to nitrate, a common endocrine disruptor (Secondi et al., 2009). Other species again alter the expression of their signals, such as males of the European starling (Sturnus vulgaris) that develop longer and more complex songs when exposed to estrogen mimics (Markman et al., 2008). The consequences these changes have for the individuals and the species, are, however, mostly unknown. The exception is endocrine disrupting chemicals that are known to interfere with species recognition, and, hence, to cause hybridizations and the merging of species. For instance two shiner species are increasingly interbreeding because of exposure to endocrine disrupting chemicals (Ward and Blum, 2012).

\section{Consequences of Altered Signals}

Changes in the habitat that alter signaling systems and influence animal communication can have further consequences for the reproductive success and viability of individuals and, hence, for the dynamics of populations. Such effects have recently gained more attention but are still poorly known. Alterations of signals can influence the reliability of the signals as indicators of mate quality, or of the resources that the mate can offer, which in turn can influence the adaptive value of mate choice. Altered mate choice can in turn influence reproductive success of individuals and, hence, population dynamics. Moreover, if the dynamics of populations change, also interactions among species may change, such as predator-prey and competitive interactions. This can in turn alter the structure and function of the species community.

Changes in signaling systems because of habitat deterioration can consequently have far reaching consequences for the viability of ecosystems and, hence, be of conservation concern. In the next sections, I will discuss the impact that altered signaling systems can have from the individual to the community level, and hence for biodiversity and the structure and function of biological communities.

\subsection{Signal Honesty}

Changes in signal expression, or in the transmission of signals through a habitat, can alter the reliability of signals as indicators of phenotypic and genetic quality. Signals have been gradually 
adjusted to environmental conditions over evolutionary time, and sudden changes in the habitat can consequently disrupt their adaptive value. For instance, increased water turbidity because of anthropogenic eutrophication reduces signal reliability in the three-spined stickleback. In clear water, males adjust their signals to their dominance status: dominant males in good condition become colorful and court at a high rate, while subdominant males fade in colors and become passive. This social control of signaling ensures honest signaling of mate quality, as dominance correlates with condition and parenting ability. However, in turbid water, the social control of signaling relaxes and males can express bright red coloration and court at a high rate independent of their condition (Wong et al., 2007).

\subsection{Reproductive Success and Population Dynamics}

Changes in signal expression, reliability or reception can in turn influence the lifetime reproductive success of individuals. Increased cost of signaling, or signal evaluation, can increase mortality risk, such as predation risk, or reduce the amount of resources available to allocate into other fitness enhancing traits, such as fecundity and parental care. Maladaptive mate choices can in turn reduce the number and viability of offspring produced. For instance, the reduced honesty of the signals of the male three-spined stickleback in turbid water, discussed in the previous section, causes females to increasing choose males that invest less in parental care and who sire offspring with a low survival probability (Candolin et al., 2016).

A fatal consequence of altered habitats are the occurrence of evolutionary traps, ie, when individuals are attracted to traits that resemble the signals used as indicators of mate quality (Schlaepfer et al, 2002). An infamous example is the jewel beetle (Julodimorpha bakewelli) that is attracted to beer bottles because the texture of the bottles resembles that of females (Gwynne and Rentz, 1983). Because the bottles also constitute supernormal stimuli - because of their large size males are more attracted to these than to females (Fig 2).

A reduction in the number or viability of offspring produced can in turn influence population growth rate. For instance, evolutionary traps could easily drive population decline. The 'traps' could even cause extinction if too many individuals are attracted to them. However, the degree to which altered signaling systems influence population dynamics is little known. An increasing number of studies show that altered efficiency of signals can cause maladaptive matings, but the ultimate impact on population viability usually remains unknown. The effect depends on the importance of sexual selection in relation to natural selection in maintaining population viability. If natural selection is strong, ie, if fecundity and juvenile mortality is high, selection may only shift from the mating stage to other life-history stages, such as the juvenile stage, which may be enough for maintaining a viable population. In contrast, if natural selection after mating is weak, ie, if few offspring are produced and juvenile mortality is low, then selection at later stages may not fully compensate for relaxed sexual selection at the mating stage, in which case maladaptive mate choice may reduce population viability.

\subsection{Species Interactions}

Habitat degradation that alters signals and communication during mate choice can influence not only interactions within the species, but also among species. In particular, changes in population dynamics because of disturbed mate choice can alter species interactions, such as predator-prey and competitive interactions. An example is avian communities disturbed by urban noise, where species that cannot adjust their vocalizations to the noise have declined, while those that can adjust have been favored (Francis et al., 2009). This has altered the composition of the species community. Such changes could cascade through the community and food web and further change these, but so far little is known about these community-wide effects

Another serious consequence of altered signals, or signal evaluation, is hindered species recognition. This can result in hybridization between species and, hence, endanger species persistence, which 
may reduce biodiversity (Seehausen, 2006). For instance, the cichlid fishes of the Great lakes of Africa have been hybridizing because of increased water turbidity, as mate choices are largely based on inter specific differences in male color patterns (Fig. 3). As a consequence, reproductive barriers among species have broken down and hybridizations occurred, which have eroded species diversity (Seehausen et al., 1997). Similarly, swordtail fishes in Mexican streams are merging into hybrid swarms due to impaired chemically mediated species recognition (Fisher et al., 2006). Such changes reduce biodiversity, with further potential consequences for the structure and function of ecosystems.

\section{Conservation implications}

Much evidence has accumulated on the detrimental effects of habitat deterioration on signaling systems, particularly in a mate choice context. However, it is worrying that little is known about the consequences of disrupted mate choice for population viability and community processes. Considering the importance that mate choice plays in ensuring viable populations, by determining the size and viability of the next generation, more attention needs to be paid to these sublethal effects of habitat deterioration. So far investigations in disturbed environments have mostly concentrated on more direct effects of habitat deterioration on wildlife diversity and viability, such as altered food availability, predation risk or disease prevalence. Yet, sublethal effects of habitat deterioration, such as disturbed communication during mate choice, could have profound effects on species persistence and the diversity of biological communities and, hence, deserve more attention in conservation work.

\section{References}

Candolin, U. (2009). Population responses to anthropogenic disturbance: lessons from three-spined sticklebacks Gasterosteus aculeatus in eutrophic habitats. Journal of Fish Biology 75, 21082121.

Candolin, U., Tukiainen, I. and Bertell, E. (2016). Environmental change disrupts communication and sexual selection in a stickleback population. Ecology 97, 969-979.

Dunlop, R. A. (2016). The effect of vessel noise on humpback whale, M egaptera novaeangliae, communication behaviour. Animal Behaviour 111, 13-21.

Fisher, H. S., Wong, B. B. M. and Rosenthal, G. G. (2006). Alteration of the chemical environment disrupts communication in a freshwater fish. Proceedings of the Royal Society B-Biological Sciences 273, 1187-1193.

Francis, C.D., Ortega, C.P. and Cruz, A. (2009). Noise pollution changes avian communities and species interactions. Current Biology. 19, 1415-1419.

Gwynne, D.T. and Rentz, D.C.F. (1983). Beetles on the bottle - Male Buprestids mistake stubbies for females (Coleoptera). J ournal of the Australian Entomological Society 22, 79-80.

Heuschele, J., Mannerla, M., Gienapp, P. and Candolin, U. (2009). Environment-dependent use of mate choice cues in sticklebacks. Behavioral Ecology 20, 1223-1227.

Leal, M. and Fleishman, L. J. (2004). Differences in visual signal design and detectability between allopatric populations of Anolis lizards. American Naturalist 163, 26-39.

Markman, S., Leitner, S., Catchpole, C., et al. (2008). Pollutants increase song complexity and the volume of the brain area HVC in a songbird. Plos One 3, 6 .

Møller, A. P. (1994). Sexual selection and the barn swallow. Oxford: Oxford University Press. 
Schlaepfer, M. A., Runge, M. C. and Sherman, P. W. (2002). Ecological and evolutionary traps. Trends in Ecology and Evolution 17, 474-480.

Secondi, J., Hinot, E., Djalout, Z., Sourice, S. and Jadas-Hecart, A. (2009). Realistic nitrate concentration alters the expression of sexual traits and olfactory male attractiveness in newts. Functional Ecology 23, 800-808.

Seehausen, O. (2006) Conservation: Losing biodiversity by reverse speciation. Current Biology. 16, 334-337.

Seehausen, O., Alphen, J. J. M . and Witte, F. (1997). Cichlid fish diversity threatened by eutrophication that curbs sexual selection. Science 277, 1808-1811.

Shenoy, K. and Crowley, P. H. (2011). Endocrine disruption of male mating signals: Ecological and evolutionary implications. Functional Ecology. 25, 433-448.

Slabbekoorn, H. and Peet, M. (2003). Ecology: Birds sing at a higher pitch in urban noise - Great tits hit the high notes to ensure that their mating calls are heard above the city's din. Nature 424, 267-267.

Sundin, J., Rosenqvist, G. and Berglund, A. (2013). Altered oceanic pH impairs mating propensity in a pipefish. Ethology 119, 86-93.

Taylor, E. B., Boughman, J. W., Groenenboom, M., et al. (2006). Speciation in reverse: morphological and genetic evidence of the collapse of a three-spined stickleback (Gasterosteus aculeatus) species pair. M olecular Ecology 15, 343-355.

Vasconcelos, R. O., Amorim, M. C. P. and Ladich, F. (2007). Effects of ship noise on the detectability of communication signals in the Lusitanian toadfish. Journal of Experimental Biology 210, 2104-2112.

Wong, B. B. M., Candolin, U. and Lindström, K. (2007). Environmental deterioration compromises socially-enforced signals of male quality in three-spined sticklebacks American Naturalist 170 , 184-189.

Ward, J.L. and Blum, M.J. (2012). Exposure to an environmental estrogen breaks down sexual isolation between native and invasive species. Evolutionary Applications 5, 901-912

\section{Further Reading}

Bradbury, R. B. and Vehrencamp, S. L. (2011). Principles of animal communication. Second edition. Sinauer Associates, Inc.

Candolin, U. and Wong, B. B. M. (2012). Behavioural responses to a changing world: mechanisms and consequences. Oxford University Press, Oxford.

Delhey, K. and Peters, A. (2017). Conservation implications of anthropogenic impacts on visual communication and camouflage. Conservation Biology 31:30-39.

Endler, J. A. (1992). Signals, signal conditions, and the direction of evolution. The American Naturalist 139, S125-S153.

Francis, C. D. and Barber, J. R. (2013). A framework for understanding noise impacts on wildlife: an urgent conservation priority. Frontiers in Ecology and the Environment 11, 305-313.

Maynard-Smith, J. and Harper, D. (2003). Animal Signals. Oxford University Press, Oxford.

Radford, A. N., Kerridge, E., and Simpson, S. D. (2014). Acoustic communication in a noisy world: can fish compete with anthropogenic noise? Behavioral Ecology 25, 1022-1030.

van der Sluijs, I. S., Gray, S. M., Amorim, M. C. P. et al. (2011). Communication in troubled waters: Responses of fish communication systems to changing environments. Evolutionary Ecology 25, 623-640.

Swaddle, J. P., Francis, C. D., Barber, J. R. et al (2015). A framework to assess evolutionary responses to anthropogenic light and sound. Trends in Ecology and Evolution 30, 550-560.

Wong, B. B. M. and Candolin, U. (2015). Behavioral responses to changing environments. Behavioral Ecology 26, 665-673. 


\section{Relevant Websites}

http://sites.sinauer.com/animalcommunication2e/index.html

http://www.nature.com/scitable/ knowledge/ library/an-introduction-to-animal-communication23648715

Figures
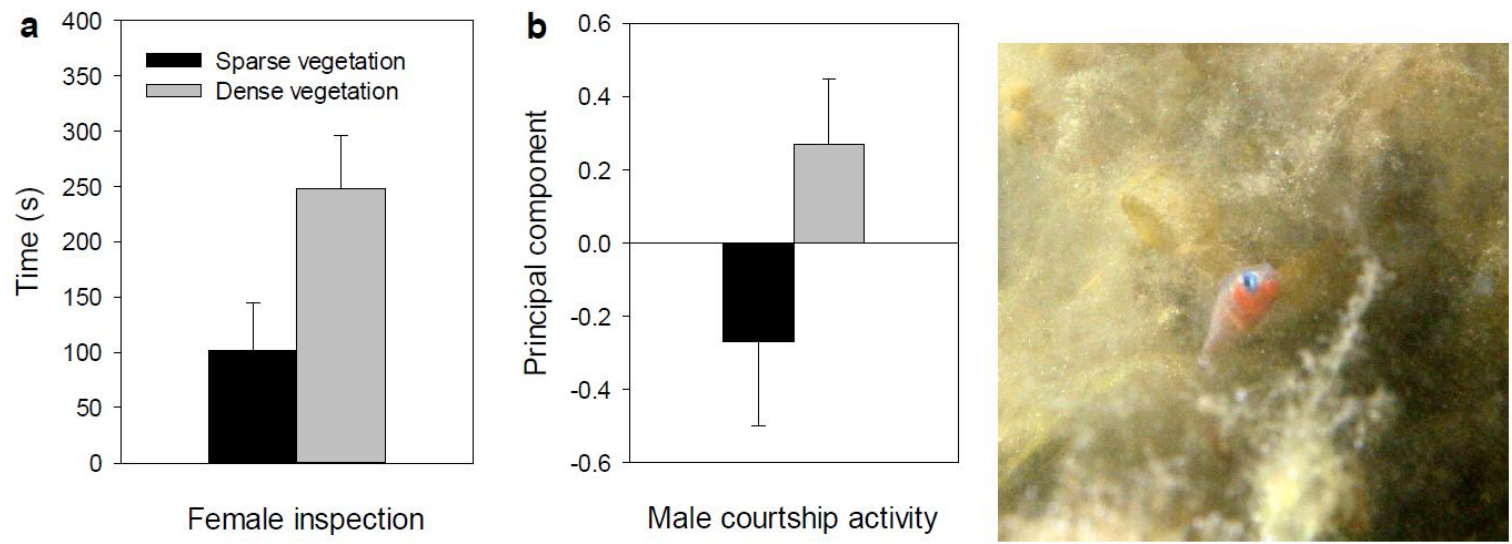

Fig. 1 When the density of filamentous algae increases because of human-induced eutrophication, female three-spined sticklebacks have to spend more time evaluating males. (b) Males in turn increase their courtship activity, but still the females have a difficult time in making their choice. Data are means + SE. Photo: Jan Heuschele

Reproduced from Candolin, U., Salesto, T., Evers, M., 2007. Changed environmental conditions weaken sexual selection in sticklebacks. Journal of Evolutionary Biology 20, 233-239. 


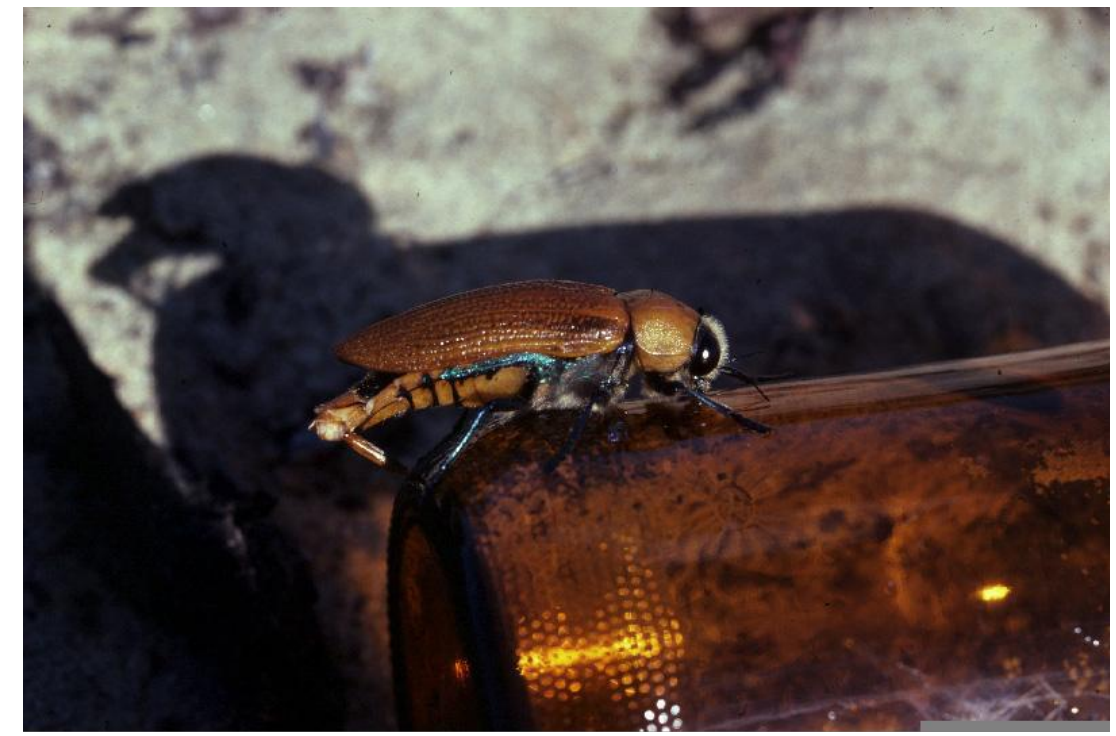

Fig. 2 A male jewel beetle (Julodimorpha bakewelli) attempting to mate with a beer bottle. Photo: Darryl Gwynne.

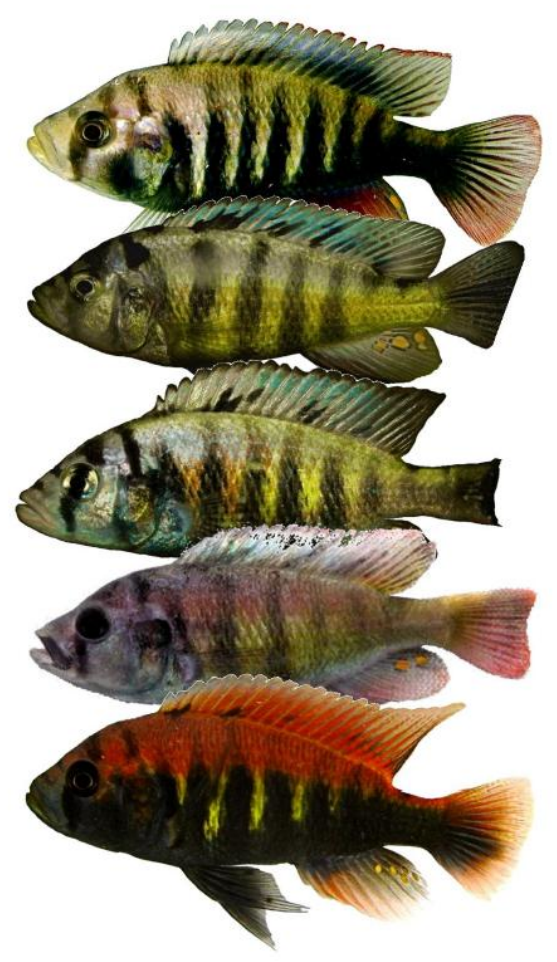

Fig. 3 Cichlids from Lake Victoria, Africa, vary in male nuptial coloration. In clear water, females mate with males of their own species based on differences in coloration. However, in turbid water, females are not able to distinguish between species based on coloration and hybrids are produced. The top and bottom images are the coloration of male Pundamilia pundamilia and Pundamilia nyererei, respectively, and the images in between are of hybrids. Photo: Ole Seehausen 